\title{
Identification of immunologic and clinical characteristics that predict inflammatory response to C. Novyi-NT bacteriolytic immunotherapy
}

\author{
Amy E. DeClue ${ }^{1 *}$ (D), Sandra M. Axiak-Bechtel ${ }^{2}$, Yan Zhang ${ }^{1}$, Saurabh Saha ${ }^{3}$, Linping Zhang ${ }^{3}$, David D. Tung ${ }^{3}$
} and Jeffrey N. Bryan ${ }^{2}$

\begin{abstract}
Background: Clostridium novyi-NT (CNV-NT), has shown promise as a bacterolytic therapy for solid tumors in mouse models and in dogs with naturally developing neoplasia. Factors that impact the immunologic response to therapy are largely unknown. The goal of this pilot study was to determine if plasma immune biomarkers, immune cell function, peripheral blood cytological composition and tumor characteristics including evaluation of a PET imaging surrogate of tumor tissue hypoxia could predict which dogs with naturally developing naïve neoplasia would develop an inflammatory response to CNV-NT.

Results: Dogs that developed an inflammatory response to CNV-NT had a higher heart rate, larger gross tumor volume, greater tumor $\left[{ }^{64} \mathrm{CU}\right.$ ATSM SUV $\mathrm{Vax}_{\text {, }}$ increased constitutive leukocyte IL-10 production, more robust NK cell-like function and greater peripheral blood lymphocyte counts compared to dogs that did not develop an inflammatory response to CNV-NT. Of these, unstimulated leukocyte IL-10 production, heart rate, and gross tumor volume appeared to be the best predictors of which dogs will develop an inflammatory response to CNV-NT.
\end{abstract}

Conclusions: Development of inflammation in response to CNV-NT is best predicted by pretreatment unstimulated leukocyte IL-10 production, heart rate, and gross tumor volume.

Keywords: Immunology, Cancer, Canine, Immunotherapy

\section{Background}

For centuries it has been recognized that certain bacterial infections could induce tumor regression. In more modern times, the controlled use of bacteriolytic immunotherapy has been evaluated in both induced and spontaneous tumor models [1]. One such proposed bacterolytic immunotherapy, Clostridium novyi-NT (CNV-NT), has shown promise as a therapy for solid tumors $[2,3]$. The advantage of CNVNT over other types of bacterolytic immunotherapy is that CNV-NT is a strict anaerobe and thus is restricted to growing in the relatively hypoxic regions of tumor tissue and not

\footnotetext{
* Correspondence: decluea@misssouri.edu

${ }^{1}$ Department of Veterinary Medicine and Surgery, Comparative Internal Medicine Laboratory, University of Missouri, College of Veterinary Medicine, 900 E. Campus Dr, Columbia, MO 65203, USA

Full list of author information is available at the end of the article
}

in healthy tissues. This should, theoretically, maximize local immune stimulation and inflammation in the tumor microenvironment while minimizing systemic effects [4].

C. novyi-NT bacteriolytic immunotherapy has successfully cured induced neoplasia in mouse models and resulted in objective tumor responses in naturally developing neoplasia in the dog [1-3]. However, the response rate in dogs with naturally developing neoplasia has been less than $40 \%$, thus strategies to optimize response rates are needed [2]. Proposed explanations for the limited response rate include a lack of appropriate immune response to infection or variability in the degree of hypoxic tissue in the tumor leading to reduced CNV-NT germination. Dogs with various forms of neoplasia have altered immune function which could impact the efficacy 
of immunotherapies like induced infection and little is known about the regional blood flow distribution in solid tumors in this species [5-8].

The purpose of this pilot investigation is to identify possible immunologic, tumor or clinical characteristics pre-treatment that would predict which dogs are more likely to successfully develop immunologically pertinent inflammation after administration of CNV-NT. To evaluate this, plasma immune biomarkers, immune cell function, peripheral blood cytological composition and tumor characteristics including evaluation of a PET imaging surrogate of tumor tissue hypoxia were measured in dogs with naturally developing naïve neoplasia prior to administration of CNV-NT; following CNV-NT treatment the development of inflammation was recorded. Baseline parameters were compared between dogs that developed inflammation and those that did not. Parameters that were significantly different between groups were then used to develop a predictive model.

\section{Methods \\ Population}

Client owned dogs presented to the University of Missouri Veterinary Health Center with histologically or cytologically confirmed soft-tissue sarcoma, oral or cutaneous malignant melanoma, oral squamous cell carcinoma, or other cutaneous carcinomas were eligible for enrollment with written informed client consent (IACUC protocol \#7386). Eligibility criteria included tumors greater than $2 \mathrm{~cm}$ in diameter, tumor size and location where surgical excision with at least marginal resection was a viable option, and body weight of $>10 \mathrm{~kg}$. Dogs were excluded if comorbidities were present that suggested survival expectation of less than 6 weeks, evidence of metastasis outside of the local draining lymph node, tumor location where abscess development would be catastrophic (e.g., CNS), persistent neutropenia or thrombocytopenia, grade 2 increases in plasma ALT, BUN or creatinine, administration of antimicrobial therapy within the 7 days preceding enrollment, concurrent infection requiring systemic antimicrobial therapy, chemotherapy, radiation therapy, or other immunotherapy within the 3 weeks preceding enrollment, pregnancy or potential pregnancy, enrollment in another clinical trial, cardiac disease severe enough that a balanced crystalloid solution administered at $4 \mathrm{~mL} / \mathrm{kg} / \mathrm{h}$ would likely induce congestive heart failure, and unavailability during the full study duration.

Baseline evaluations included medical history, physical examination, complete blood count, plasma biochemical profile, complete urinalysis, thoracic radiographs, and cytologic evaluation of the draining lymph bed if accessible. Prior to therapy, tumors were evaluated using PET/CT to evaluate tumor size, estimate glucose metabolism with $\left[{ }^{18} \mathrm{~F}\right] \mathrm{FDG}$, and estimate relative tumor hypoxia using $\left[{ }^{64} \mathrm{Cu}\right]$ ATSM (some data not presented in this manuscript).

\section{Treatments}

Dogs received CNV-NT (BioMed Valley Discoveries Inc., Kansas City, MO) either intravenous (IV) or intratumoral (IT) on day 0 . The change from IV to IT was based on a programmatic decision by the sponsor.

Intravenous administration CNV-NT $\left(1 \times 10^{9}\right)$ spores were administered in $50 \mathrm{mls}$ of physiologic saline through an intravenous catheter. Initially, $5 \mathrm{~mL}$ was administered over 2 min; dogs' vital parameters were monitored for $30 \mathrm{~min}$. If the dog's vital parameters were within $10 \%$ of baseline after $30 \mathrm{~min}$, the remaining $45 \mathrm{~mL}$ was administered over a $5 \mathrm{~min}$ time period. Following completion of CNV-NT infusion, intravenous fluids were administered for $2 \mathrm{~h}$ and dogs were discharged to their owners if no signs of a reaction was noted.

Intratumoral administration $\mathrm{CNV}-\mathrm{NT}$ was provided in $3 \mathrm{~mL}$ of saline at a dose of $1 \times 10^{8}$ spores. Following sedation, the tumor was clipped and aseptically prepped, and $\mathrm{CNV}$-NT was administered directly into the tumor with $0.75 \mathrm{~mL}$ injected into 4 total sites evenly distributed within the tumor. Vital parameters were monitored for $3 \mathrm{~h}$ following injection.

\section{Groups}

The primary outcome measure used to group the dogs was the development of clinically identifiable inflammation within 28 days of treatment for the IV group and within 56 days of treatment for the IT group. In the IV CNV-NT group, dogs that developed fever (rectal temperature $\left.>39.2^{\circ} \mathrm{C}\left(102.5^{\circ} \mathrm{F}\right)\right)$ and lethargy were placed in the inflammation group while dogs that developed no clinical signs following infusion were classified as having not developed inflammation. In the IT group, dogs that developed clinical evidence of abscess formation including edema, erythema, and/ or mucopurulent discharge were placed in the inflammation group. Dogs that had no evidence of abscess formation were placed in the no evidence of inflammation group.

\section{Sample collection and processing}

At baseline (pre-treatment), blood was collected from the jugular vein and placed in either EDTA, sodium heparin, lithium heparin or evacuated tubes for immunologic evaluation. Lithium heparinized blood was immediately cooled and centrifuged within 1 hour of collection. Plasma or serum was collected and stored at $-80{ }^{\circ} \mathrm{C}$ for batch analyses of immune protein evaluation. The remainder of the blood was processed immediately for $\mathrm{PBMC}$ isolation, $\mathrm{CBC}$, phagocytic function and leukocyte cytokine production assays as indicated below. 


\section{Clinical/tumor characteristics}

Dogs were evaluated prior to therapy utilizing rectal temperature, heart rate, complete blood count, plasma globulin concentration (total protein- albumin = globulin), CT scan measurement of tumor volume and PET scan with $\left[{ }^{18}\right.$ F]FDG to characterize tumor metabolism and $\left[{ }^{64} \mathrm{Cu}\right]$ ATSM to characterize tumor hypoxia. $\left[{ }^{64} \mathrm{Cu}\right] \mathrm{ATSM}$ is reduced and trapped in hypoxic cells, localizing for imaging with positron emission tomography (PET). Dogs were injected with $74 \mathrm{MBq}(2 \mathrm{mCi})$ of $\left[{ }^{64} \mathrm{Cu}\right] \mathrm{ATSM} 45 \mathrm{~min}$ prior to scanning. Dogs were anesthetized and maintained on a propofol infusion breathing room air. Scans were acquired with a Philips C-PET+ with attenuation correction. Reconstructed images were evaluated using MIMfusion software. Maximum standard uptake value $\left(\mathrm{SUV}_{\mathrm{Max}}\right)$ and threshold volume with $\mathrm{SUV}>1.0$ were calculated.

\section{Plasma immune biomarkers}

Plasma was collected centrifuged at $400 \mathrm{~g}$ for $15 \mathrm{~min}$ and then stored at $-80{ }^{\circ} \mathrm{C}$ for batch analysis. Thirteen immune markers: IL-6, CXCL-8, IL-2, IL-7, TNF- $\alpha$, GM-CSF, IL18, CXCL-1, IP-10, IL-15, IL-10 and MCP-1 were evaluated in undiluted plasma as previously described using canine specific multiplex bead-based, ELISA assay (Millipore Sigma, Burlington, MA), a MAGPIX Multiplexing instrument and analyzed using MILLPLEX Analyst 5.1 software [9]. Serum HMGB-1 (IBL International, Toronto, ON) and CRP (ICL Inc., Portland OR) were measured using commercially available ELISAs according to the manufacturers' recommendations $[10,11]$.

\section{Immune cell function}

Immune cell function was assessed by evaluating granulocyte phagocytic and respiratory burst function, leukocyte cytokine production capacity and NK-like cell function.

Phagocytic function was determined using the Phagotest ${ }^{\oplus}$ commercial test kit (Orpegen Pharma, Heidelberg, Germany) which evaluates phagocytosis of FITC-labeled, opsonized E. coli. The assay has been previously validated in dogs and was performed as previously described $[5,7]$. Samples were analyzed by flow cytometry using the CyAn ADP flow cytometer (Beckman Coulter, Brea, CA) and associated data analysis software (Summit V 5.2.0.7477, Brea, CA) within $30 \mathrm{~min}$ and a minimum of 15,000 events were recorded for each sample. DNA stain positive cells were gated and placed on a forward and side scat plot. Phagocytes were identified using standard forward and side scatter characteristics. Then, FITC positive phagocytes were identified on a histogram. Both the relative number of $E$. coli-positive cells as well as the mean fluorescence intensity (MFI) of positive cells indicating the number of bacteria per cell were recorded.
The quantification of oxidative burst of PMNs was performed using a Phagoburst ${ }^{\ominus}$ kit (ORPEGEN Pharma, Heidelberg, Germany) which evaluates E.coli and PMA-induced oxidative burst using dihydrorhodamine 123 as a fluorogenic substrate. The assay has been previously validated in dogs and was performed as previously described $[5,7]$. Samples were analyzed by flow cytometry using the CyAn ADP flow cytometer and associated data analysis software within $30 \mathrm{~min}$ and a minimum of 15,000 events were recorded for each sample. DNA stain positive cells were gated and placed on a forward and side scatter plot. Phagocytes were identified using standard forward and side scatter characteristics. Then, a FLI histogram was used to identify positive phagocytes. The percentage of positive cells indicating recruitment and the MFI indicating the intensity of oxidative burst were recorded.

Leukocyte cytokine production capacity was determined by stimulating whole blood with lipopolysaccharide (LPS) from Escherichia coli 0127:B8 (final concentration, $100 \mathrm{ng} \mathrm{mL}^{-1}$; Sigma-Aldrich, St. Louis, MO), lipoteichoic acid (LTA) from Streptococcus faecalis (final concentration, $1000 \mathrm{ng} \mathrm{mL}^{-1}$; Sigma-Aldrich), or phosphate buffered saline (PBS; unstimulated control) and then measuring cytokine concentrations in the cell culture supernatant as previously described [12]. Blood was diluted 1:2 with media and samples were cultured on 12 well plates with LPS, LTA or PBS and then incubated for $24 \mathrm{~h}$ at $37^{\circ} \mathrm{C}$ in $5 \% \mathrm{CO}_{2}$. Cell supernatant was collected at end of incubation and stored in $-80{ }^{\circ} \mathrm{C}$ for analysis. Quantification of TNF- $\alpha$, IL-6 and IL-10 was accomplished using a canine specific multiplex bead-based, ELISA assay (Millipore Sigma) and a MAGPIX Multiplexing instrument as stated for the plasma immune markers.

NK-like cell function was determined using a thyroid adenocarcinoma cytotoxicity assay as previously described [8]. Canine thyroid adenocarcinoma (CTAC) cells were used as target cells. Prior to the assay, CTAC cells were labeled with $3 \mathrm{mM}$ green fluorescent 3,3'-Dioctadecyloxacarbocyanine $(\mathrm{DiO})$ for $20 \mathrm{~min}$ at $37{ }^{\circ} \mathrm{C}$ in $5 \% \mathrm{CO}_{2}$. Cytotoxicity of cancer cells was assessed by co-incubating PBMC with $\mathrm{DiO}-$ labeled CTAC cells for $24 \mathrm{~h}$ at $37{ }^{\circ} \mathrm{C}$ with $5 \% \mathrm{CO}_{2}$. Cells were comingled in different PBMC to Dio-CTAC cell ratios: 1:1, 10:1, 25:1 and 50:1. Single cell population of PBMC or Dio-CTAC were used as controls. At end of incubation, cells were incubated with propidium iodide (PI). Samples were analyzed using the CyAn ADP flow cytometer and associated data analysis software. A minimum of 10,000 events were recorded for each sample. Data were analyzed as previously described [8]. Briefly, the CTAC cells were gaited on a forward/ side scatter plot and then applied to a plot comparing $\mathrm{DiO}$ and PI. DiO and PI positivity were determined using unstained cells as controls. Cells positive for $\mathrm{DiO}$ and PI were defined as dead CTAC cells. Baseline cell death was established using DiO/PI stained CTAC cells alone. The NK-like 
cell killing index was calculated by dividing the \% death from the PBMC+CTAC cell mixture by the CTAC cells alone.

\section{Peripheral blood immune cell composition}

Peripheral blood immune cell composition was determined by performing a complete blood count to determine the number of total white blood cells, neutrophils, monocytes and lymphocytes in the peripheral blood and flow cytometry to determine lymphocyte phenotype. A complete blood count was performed by the University of Missouri Veterinary Diagnostic Laboratory. Antibodies used for PBMC phenotype assay were rat anti-mouse CD3-PE (abcam, Cambridge, UK; Clone KT3), mouse anti-dog CD21-Alexa Fluor 647 (AbD Serotec, Raleigh, NC; Clone: CA2.1D6), rat anti-mouse/rat FoxP3-APC (eBioscience, San Diego, CA; Clone: FJK-16 s), rat-anti-dog CD4-FITC (AbD Serotec; Clone: YKIX302.9), mouse anti-human CD25-PE (Dako; Clone: ACT-1), rat anti-human CD3-Alexa Fluor 647 (AbD Serotec; Clone: CD3-12), rat anti-dog CD8-APC (eBioscience; Clone: YCATE55.9), anti-human CD56-PE (Dako, Santa Clara, CA; Clone: MOC-1), Rat IgG2a APC isotype control, Mouse IgG1 PE isotype control and Rat IgG2a FITC isotype control (eBioscience). PBMCs were harvested and $200 \mu \mathrm{l}$ of $10^{6}$ PBMC per sample were added to a 96 well round bottom plate. Plates were centrifuged at $300 \mathrm{~g}$ for $6 \mathrm{~min}$ and supernatant removed. $50 \mu \mathrm{l}$ of FACS buffer was added to each sample and then samples were stained for the following markers: CD4 T cell $\left(\mathrm{CD}^{+} / \mathrm{CD}^{+}\right)$, CD8 $\mathrm{T}$ cell $\left(\mathrm{CD}^{+} / \mathrm{CD}^{+}\right)$, NK-like cell $\left(\mathrm{CD}^{-} / \mathrm{CD} 56^{+}\right)$and $\mathrm{B}$ cell $\left(\mathrm{CD} 21^{+}\right)$for $30 \mathrm{~min}$ on ice protected from light. At end of incubation cells were washed twice with PBS and fixed with $2 \%$ paraformaldehyde. $\mathrm{T}$ regulatory cells $\left(\mathrm{CD} 4^{+} / \mathrm{CD} 25^{+} / \mathrm{FoxP}^{+}\right)$were detected using surface CD4 and CD25 stains and incubating for $30 \mathrm{~min}$ as previously described [13]. Cells were washed with PBS, then fixed and permeabilized with fix/perm FoxP3 staining buffer (eBioscience) for $30 \mathrm{~min}$ to detect intracellular FoxP3. At the end of incubations, cell were washed and collected into FACS tubes; all samples were then analyzed at the University of Missouri Cell and Immunology Core Facility using CyAn ADP and Summit software (Summit V 5.2.0.7477, CA, USA). A minimum of 15,000 events were recorded for each sample. Unstained cells and matched isotype controls from the same manufacturer were used for negative antibody controls for analyses. Lymphocytes were identified and gated using a forward and side scatter plot. To evaluate $\mathrm{CD}^{+} / \mathrm{CD}^{+}$or $\mathrm{CD}^{+} / \mathrm{CD}^{+} \mathrm{T}$ cells, the gated lymphocytes were then applied to PE (CD3) vs FITC (CD4) or PE (CD3) vs APC (CD8) plots, respectively, and double positive cells were identified (Fig. 1).

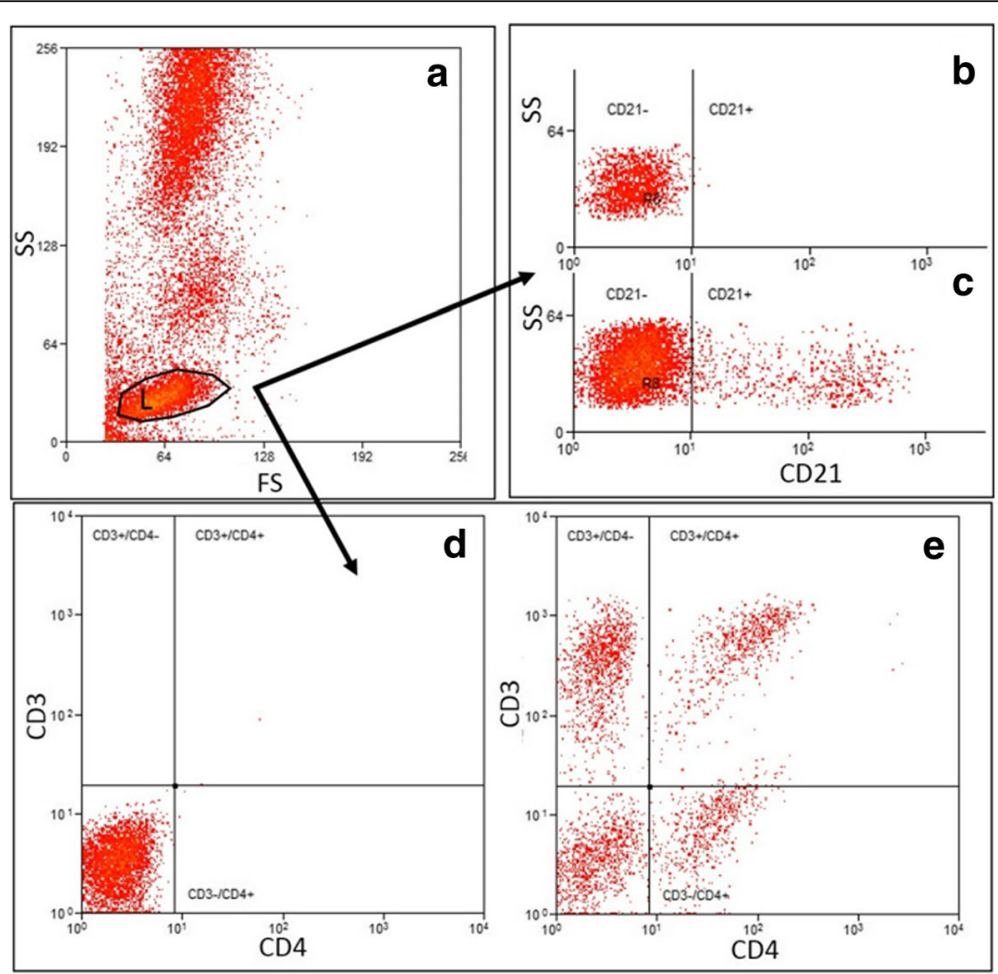

Fig. 1 Example gating scheme for identification of $\mathrm{CD}^{+} / \mathrm{CD} 4^{+}$lymphocytes and $\mathrm{CD} 21^{+}$lymphocytes. Lymphocytes were identified and gated using a forward and side scatter plot (a). Gated lymphocytes were applied were then applied to PE (CD3) vs FITC (CD4) plots or an Alexa Fluor 647 (CD21) histogram, respectively. Unstained cells and matched isotype controls from the same manufacturer were used to determine cut offs for negative ( $\mathbf{b}$ and $\mathbf{d}$ ) versus positive ( $\mathbf{c}$ and $\mathbf{e}$ ) cells. $\mathrm{CD}^{+} / \mathrm{CD}^{+}$or $\mathrm{CD} 21$ positive cells were then identified. $\mathrm{CD}^{+} / \mathrm{CD}^{+}$cells were selected in the same manner as the $\mathrm{CD}^{+} / \mathrm{CD}^{+}$cells 
To identify B cells, lymphocytes were applied to an Alexa Fluor 647 (CD21) histogram (Fig. 1). NK-like cells were identified by applying the gated lymphocytes to an Alexa Fluor 647 (CD3) vs side scatter plot and selecting for the Alexa Fluor 647 negative cells. The CD3 negative cells were then applied to a PE (CD56) vs side scat plot and the PE positive cells were selected (Fig. 2). T regulatory cells were identified by applying the gated lymphocytes to a FITC (CD4) vs PE (CD25). Then FITC and PE double positive cells were applied to an APC (Fox P3) vs side scatter plot as previously described [13].

\section{Statistical analysis}

Statistical analysis was accomplished using SigmaPlot software (SigmaStat; Systat Software Inc., San Jose, CA). To construct the initial model, variables were compared using a univariate analysis to identify parameters that differed between dogs that developed inflammation and those that did not. A Mann-Whitney Rank Sum test was used in initial comparison of variables between groups. Because the goal of this pilot investigation was to identify markers that would predict which dogs developed inflammation at the tumor site and we did not want to inappropriately exclude markers due to type II statistical error, a $p$-value of $<0.10$ was considered statistically significant and variables with a $p$-value less than this cut-off were included in predictive modeling. Parameters that were significantly associated with the development of inflammation were then entered into a best subsets regression model. Best subsets were developed based on $\mathrm{R}$ squared as the best criterion. The final model selection was determined by selecting the model with the greatest $\mathrm{R}$ squared with the smallest Mallows $\mathrm{Cp}$ value, significant contribution from all variables $(p<0.05)$ and minimal multicollinearity based on a variance inflation factor of $<1.50$. Data are presented as median and range unless otherwise noted.

\section{Results}

\section{Patient population}

Twenty dogs were enrolled, 10 dogs received IV and 10 dogs received IT administration of CNV-NT. The median age of dogs enrolled was 9.5 years (range 5-13 years, age of one dog unknown) and the median weight was $28.2 \mathrm{~kg}$ (range 14.4-70 kg). The sexes included intact male $(n=2)$, neutered male $(n=10)$, and spayed female $(n=8)$. Breeds of dogs enrolled were Labrador retriever $(n=5)$, Golden retriever $(n=3)$, Husky $(n=3)$, and one each of the following breeds: Standard Poodle, American Staffordshire terrier, English setter, Pug, Beagle, Bloodhound, Border Collie, Pointer, and mixed breed. Tumor types were soft tissue sarcoma (STSA) $(n=10)$, oral sarcoma $(n=6)$, oral melanoma $(n=3)$, and carcinoma $(n=1)$. One dog in the IV group was excluded from analysis due to development of gastric dilation and volvulus 9 days post-infusion, necessitating surgery and subsequent antibiotic therapy. This dog was withdrawn from trial and 19 dogs were included in the immunologic analysis. Of the dogs with evaluable data, 14 were classified as having developed inflammation, six in the IV group and eight in the IT group; 5 did not develop

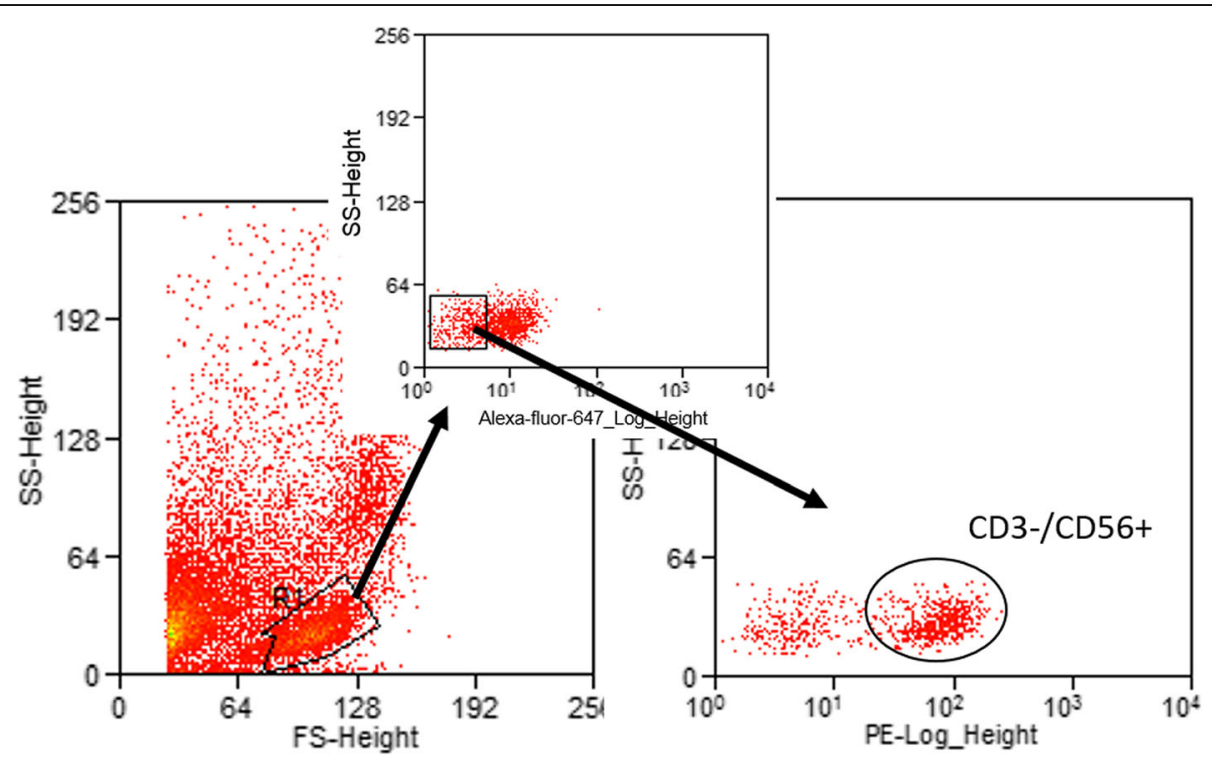

Fig. 2 Example gating scheme for identification of CD3-/CD56+ lymphocytes. Lymphocytes were identified and gated using a forward and side scatter plot. Gated lymphocytes were applied to an Alexa Fluor 647 (CD3) vs side scatter plot and selecting for the Alexa Fluor 647 negative cells. Then PE positive cells were selected on a PE (CD56) vs side scat plot 

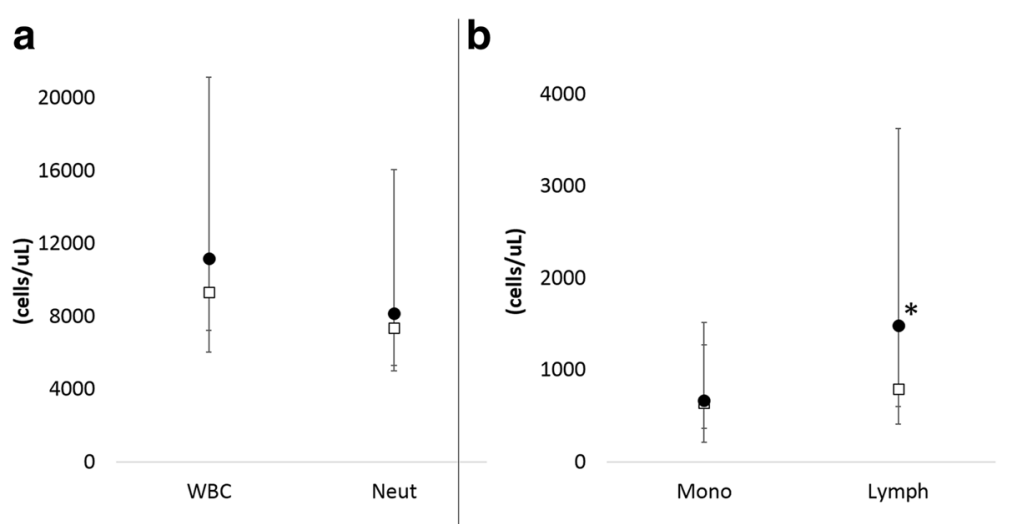

Fig. 3 Comparison of median and range of (a) peripheral blood white blood cell (WBC), neutrophil (Neut), (b) monocyte (Mono) and lymphocyte (Lymph) counts between dogs that developed inflammation (closed circles) and those that did not (open squares). ${ }^{*} p=0.087$

inflammation. There was no difference in body weight between the groups.

\section{Clinical/Tumor parameters}

Peripheral blood white blood cell, neutrophil and monocyte counts did not differ between groups (Fig. 3). However, peripheral blood lymphocyte count was significantly greater in the dogs that developed inflammation compared to those that did not $(p=0.087)$. Heart rate was significantly higher in dogs that developed inflammation compared to those that did not (Fig. $4, p=0.055$ ). Gross tumor volume was significantly larger for dogs that developed inflammation compared to those that did not (Fig. $4, p=0.058$ ). $\left[{ }^{64} \mathrm{Cu}\right]$ ATSM scan $\mathrm{SUV}_{\mathrm{Max}}$ was significantly greater for dogs that developed inflammation (Fig. $4, p=0.092$ ), but there was no difference in the threshold volume of tissue with SUV $>1.0$ between groups. There was no difference in initial rectal temperature between groups (data not shown).

\section{Serum/plasma immune biomarkers}

Plasma concentrations of IL-6, IL-2, IL-7, GM-CSF, IL18, IL-15, MCP-1, TNF- $\alpha$, IL-10 and IP-10 fell below the lower limit of detection for $\geq 50 \%$ of the samples and thus were not statistically analyzed (data not shown). Serum HMGB-1 and CRP and plasma CXCL-8 and CXCL-1 concentrations were not significantly different between groups (Fig. 5).

\section{Immune cell function}

Unstimulated leukocyte IL-10 (Fig. 6, $p=0.060$ ) production was significantly greater in the dogs that developed an inflammatory response compared to those that did not. Unstimulated leukocyte TNF- $\alpha$ and IL- 6 production and LPS or LTA-stimulated leukocyte TNF- $\alpha$, IL- 6 and IL-10 production did not significantly differ between groups (Fig. 6). NK cell function was significantly greater in the dogs that developed an immune response compared to those that did not (Fig. 7, $p=0.043$ ). There was no difference in the percentage of cells undergoing phagocytosis or the number of bacteria phagocytized, nor was there a
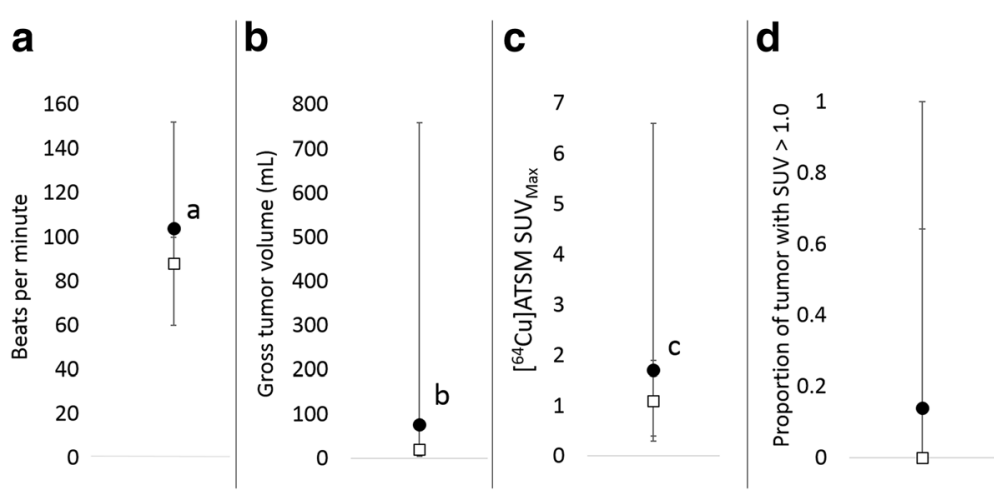

Fig. 4 Comparison of median and range of heart rate (a), gross tumor volume (b), $\left[{ }^{64} \mathrm{Cu}\right] A \operatorname{ATSM~SUV}_{\text {Max }}(\mathbf{c})$ and threshold volume of tissue with SUV $>1.0$ (d) between dogs that developed inflammation (closed circles) and those that did not (open squares). ${ }^{a} p=0.055 .{ }^{b} p=0.058,{ }^{c} p=0.092$ 

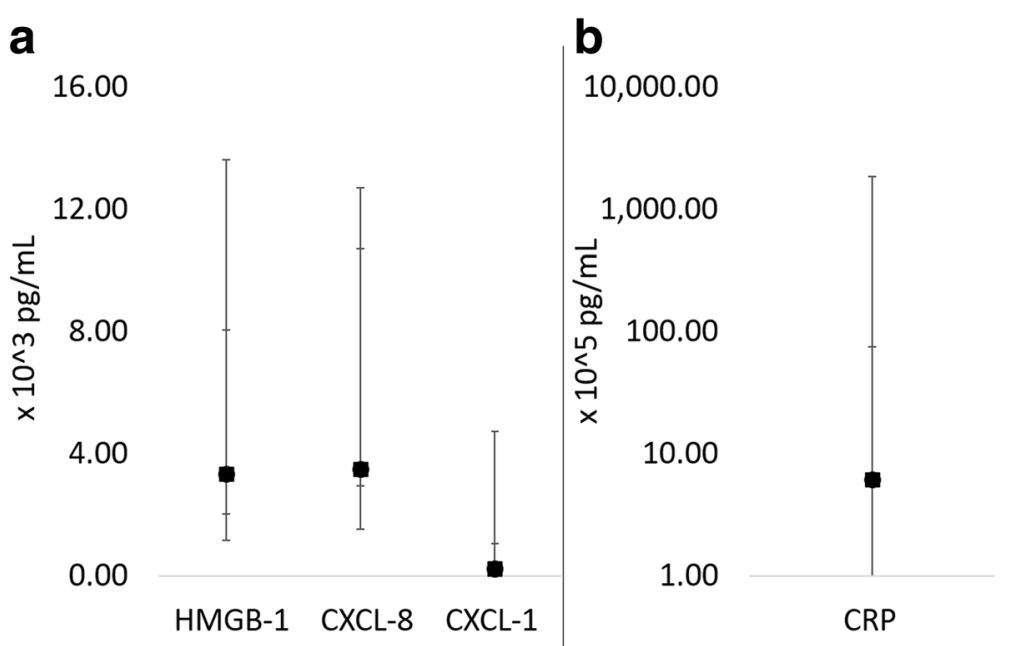

Fig. 5 Comparison of median and range of serum (a) HMGB-1 and (b) CRP and plasma (a) CXCL-8 and (a) CXCL-1 concentrations between dogs that developed inflammation (closed circles) and those that did not (open squares). Please note that CRP is on a log scale. There were no significant differences between groups

difference in the percentage of cells performing oxidative burst stimulated by E. coli or PMA or the intensity of the oxidative burst between groups (Fig. 8).

\section{Peripheral lymphocyte composition}

While peripheral blood lymphocyte count was significantly greater in the dogs that developed inflammation compared to those that did not, there were no differences in peripheral blood lymphocyte phenotype (CD4, CD8, CD21, Treg) between groups (Fig. 9).

\section{Predicting inflammation}

Best subsets regression was used to evaluate the variables unstimulated leukocyte IL-10 production, leukocyte NKlike activity, heart rate, lymphocyte count, gross tumor volume and $\left[{ }^{64} \mathrm{Cu}\right] \mathrm{ATSM}$ scan $\mathrm{SUV}_{\mathrm{Max}}$ max for prediction of inflammation. The model containing a combination of unstimulated leukocyte IL-10 production, heart rate, and gross tumor volume appeared to be the best predictor of inflammation (Adjusted R squared 0.553; Cp 2.405; $p<$ $0.034)$.

\section{Discussion}

Dogs that developed an inflammatory response to CNVNT had a higher heart rate, larger gross tumor volume, greater tumor $\left[{ }^{64} \mathrm{Cu}\right]$ ATSM SUV $\mathrm{SUx}_{\text {Max }}$ increased constitutive leukocyte IL-10 production, more robust NK cell-like function and greater peripheral blood lymphocyte counts compared to dogs that did not develop an inflammatory response to CNV-NT. Of these, unstimulated leukocyte

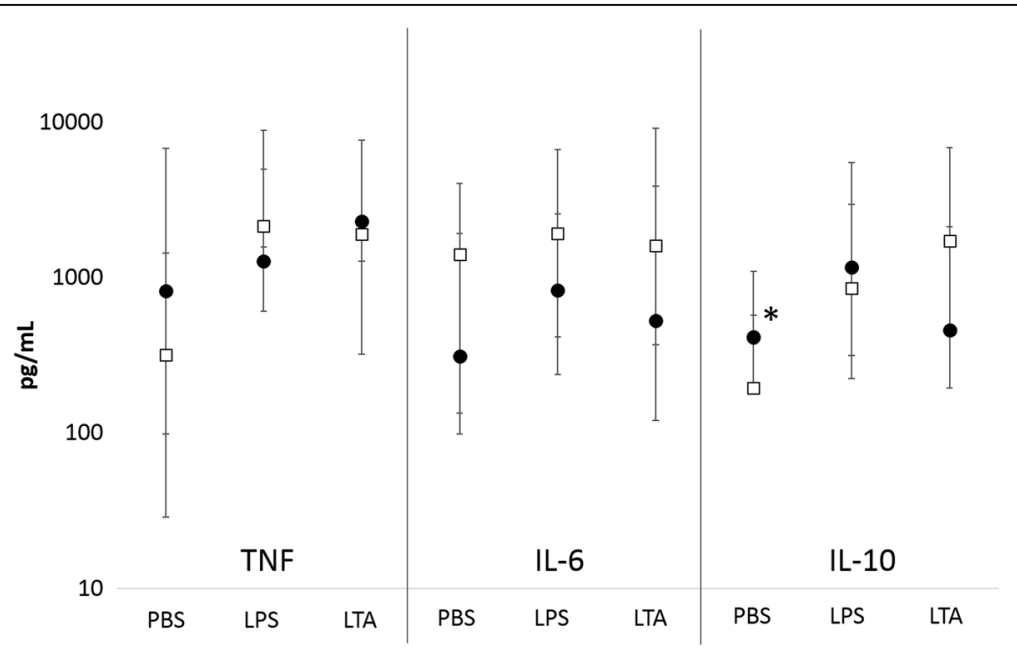

Fig. 6 Comparison of median and range for PBS (control, unstimulated), lipopolysaccharide (LPS) and lipoteichoic acid (LTA) stimulated TNF-a, IL6 and IL-10 on a logarithmic scale between dogs that developed inflammation (closed circles) and those that did not (open squares). ${ }^{*} p=0.060$ 


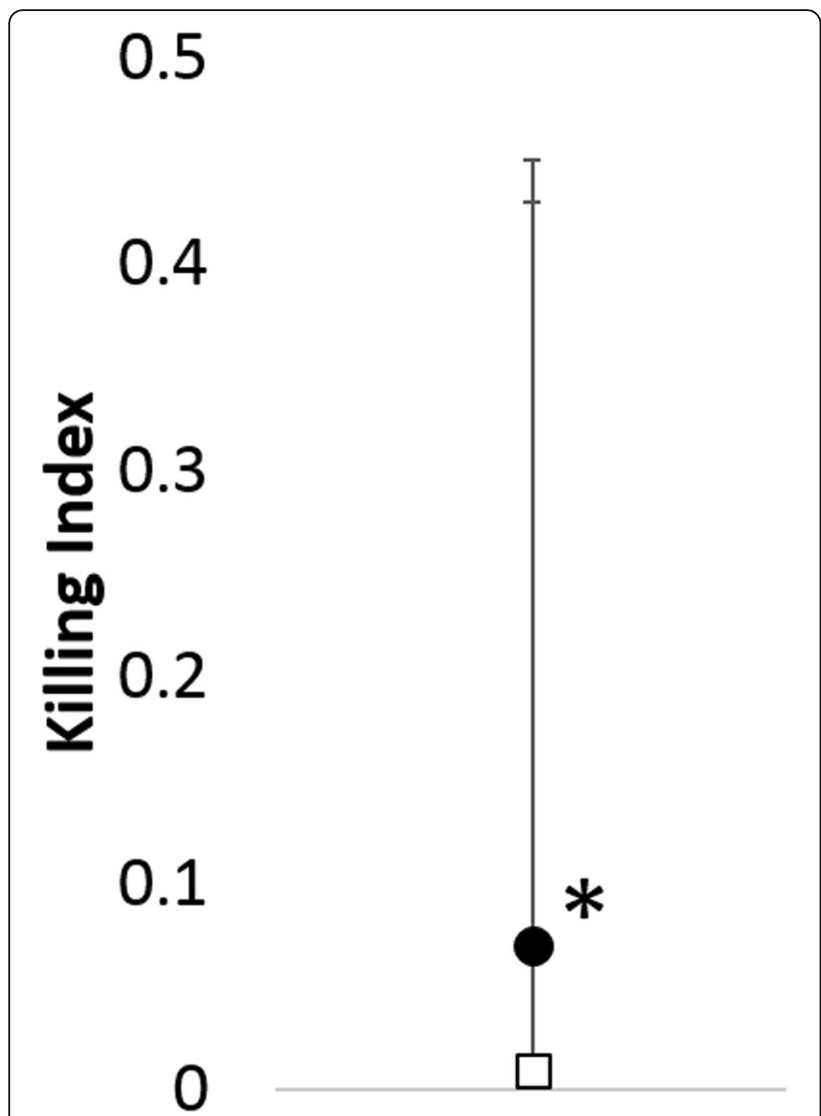

Fig. 7 Comparison of median and range for NK-like cell function killing index between dogs that developed inflammation (closed circles) and those that did not (open squares). ${ }^{*} p=0.043$

IL-10 production, heart rate, and gross tumor volume appeared to be the best predictors of which dogs will develop an inflammatory response to CNV-NT.

Ideally, we would use routinely measured clinical parameters to identify which dogs are more likely to have an inflammatory response to CNV-NT. In this study, we evaluated clinical and tumor characteristics including gross tumor volume, $\left[{ }^{64} \mathrm{Cu}\right]$ ATSM uptake, heart rate, rectal temperature, hematocrit, peripheral white blood cell count and plasma globulin concentrations as possible clinical biomarkers. However, only heart rate, peripheral leukocyte cell count, $\left[{ }^{64} \mathrm{Cu}\right]$ ATSM uptake and gross tumor volume were significantly different between groups. Of these, heart rate and gross tumor volume were predictors of development of inflammation in response to CNV-NT administration in our best subsets model. Tachycardia could be caused by activation of the sympathetic nervous system which has been associated with more severe inflammatory responses to infection. This might explain why dogs that developed inflammation had higher heart rates. It has traditionally been expected that as tumor volume increases, the relative percentage of hypoxic tissue increases. Germination of CNV-NT appears to be more robust in tumors with larger volumes and greater hypoxic regions [14]. Therefore, it was expected that a greater $\left[{ }^{64} \mathrm{Cu}\right]$ ATSM SUV $\mathrm{Max}$ and larger tumor volume would be associated with greater germination and a more intense inflammatory response.

Failure to generate an appropriate immune response to infection could impact the efficacy of CNV-NT bacteriolytic immunotherapy. Immunodysfunction has been identified in dogs and people with cancer. Altered neutrophil phagocytosis, respiratory burst function, cytokine production, peripheral circulating lymphocyte phenotype distribution and NK-cell like function have all been reported in dogs and people with cancer [5-8]. Whether these changes represent a paraneoplastic syndrome or primary immunodysfunction is unclear and it is likely that both mechanisms are observed in the dog and human population. In evaluating baseline immune system characteristics, we found that constitutive leukocyte IL10 production, NK cell-like function and greater peripheral blood lymphocyte counts were significantly different

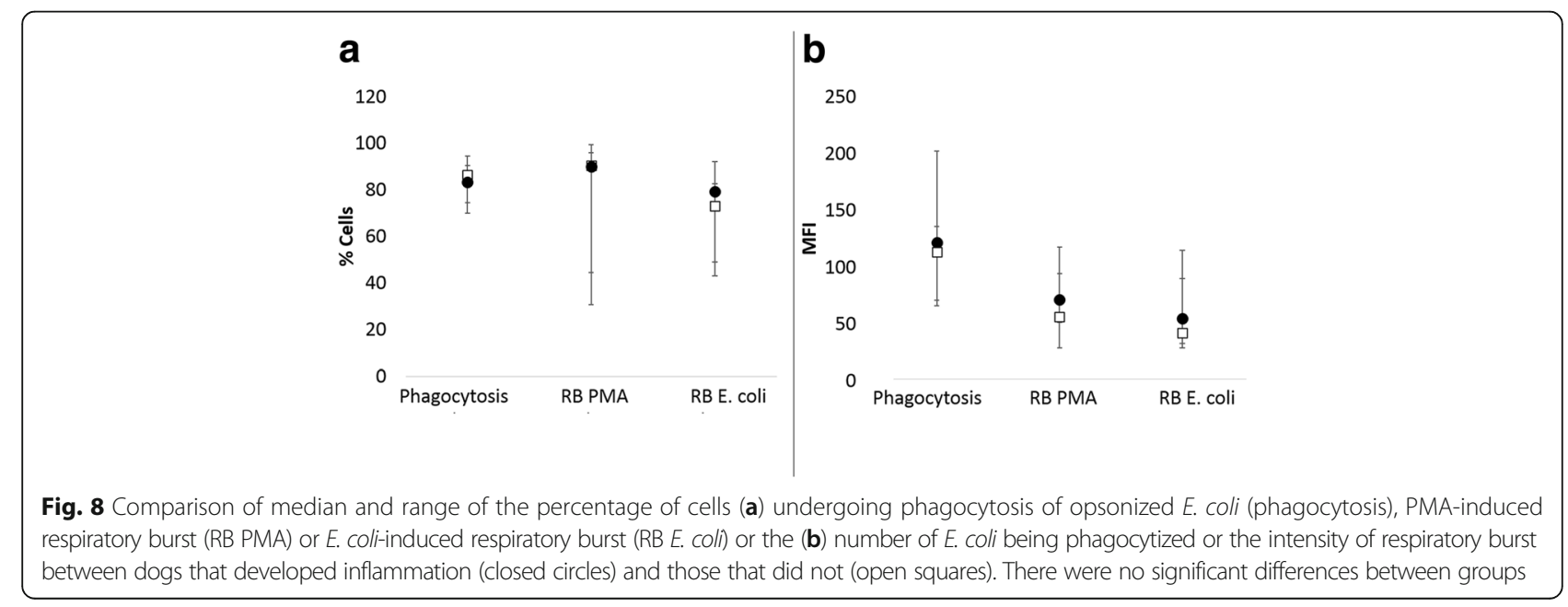




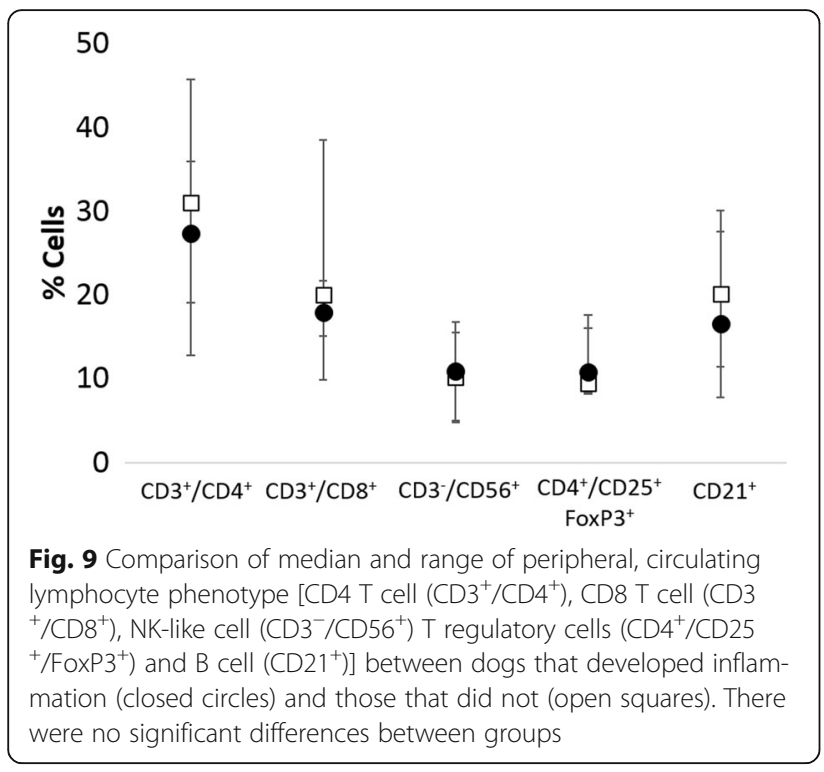

between dogs that developed inflammation in response to CNV-NT and those that did not. Of these, only constitutive leukocyte IL-10 production was selected as a predictor of development of inflammation in response to CNV-NT administration in our best subsets model. This was an unexpected finding since production of IL-10 is an immunosuppressive cytokine that has been implicated in cancer-associated immunodysfunction. It is possible that dogs with greater constitutive IL-10 production were immunosuppressed at the time of CNV-NT administration. This immunosuppression allowed CNV-NT germination to go unchecked by the immune system initially, allowing for a larger bacterial dose than dog with less IL-10 and a competent immune system. This greater bacterial dose eventually provided enough stimulus to overcome the immunosuppression and allow for the measurable inflammatory response.

We identified differences in NK cell-like function and peripheral blood lymphocyte counts between the dogs that developed inflammation and those that did not, however, these characteristics were not identified as good predictors of the development of inflammation. Stress-induced lymphopenia is a common finding in clinically ill dogs. This physiologic response is mediated by glucocorticoids, which also have direct immunosuppressive effects. It is possible that dogs with lymphopenia had greater circulating concentrations of glucocorticoids which resulted in suppression of the inflammatory response to CNV-NT. NK-like cells are important in anti-tumor and cytotoxic immune responses. NK-cells are also thought to be important effector cells during clostridium infection [15]. Greater baseline NK-like cell function might have allowed for a greater immune response to CNV-NT germination.
Larger-scale studies focused on the impact of stress hormones and baseline NK-like cell function are needed to tease out the importance of these markers.

There were several limitations to this study. First, this was a pilot study and therefore the number of dogs enrolled was limited and the cut-off point for determining significance was a $p<0.10$. The rationale for this was to avoid type II statistical error and insure that any possible candidate biomarker would be identified to assist with development of future studies in a larger patient population. We also did not compare difference between IV and IT administration. Prior to clinical use of these biomarkers, they must be studied in a larger population and comparisons made between IV and IT administration. We did not correlate induction of inflammation with tumor response or with definitive germination of $\mathrm{CNV}$ NT in this study, but this is needed to understand the full importance of these biomarkers. Further, while this study focused on solid tumors, there were three histologic tumor types in multiple anatomic locations. It is possible that this heterogeneity impacted our results. The lower limit of detection of the plasma immune markers are relatively high due to the relative analytical insensitivity of the currently available assays for dogs. In the future, more analytically sensitive assays should be developed.

\section{Conclusion}

In this pilot study, we identified several parameters that might help identify dogs with cancer that are more likely to have an inflammatory response to CNV-NT. In the future, investigators should consider evaluating if heart rate, tumor volume, surrogate markers of tumor hypoxia, IL-10 production, NK-like cell function and circulating lymphocyte count could be useful markers for patient selection or stratification of patients in clinical trials evaluating $\mathrm{CNV}-\mathrm{NT}$ for the treatment of cancer.

\section{Acknowledgements \\ The authors thank Juliana Amorim and Debbie Tate for their technical assistance. \\ Funding \\ Funding was provided by Biomed Valley Discoveries. Biomed Valley Discoveries provided monetary support and NT-CNV spores. Some of the authors (SS, LZ, DT) are employed by Biomed Valley Discoveries. These individuals contributed to the initial study design, interpretation of the data analysis and final manuscript review.}

\section{Availability of data and materials}

The datasets used and/or analysed during the current study are available from the corresponding author on reasonable request.

\section{Authors' contributions}

$A D$ developed the study design, analyzed and interpreted the data and was a major contributor in writing the manuscript. SB developed the study design, managed the clinical trial, analyzed and interpreted the data and was a contributor in writing the manuscript. $Y Z$ performed the immunologic assays and interpreted the data. SS, LZ and DT developed the study design 
and interpreted the data. JB developed the study design, managed the clinical trial, and analyzed and interpreted the data. All authors read, edited and approved the final manuscript.

\section{Ethics approval and consent to participate}

This study was approved by the University of Missouri Animal Care and Use Committee (protocol \#7386). Written consent was obtained from the animal owners prior to enrollment.

\section{Consent for publication}

NA

\section{Competing interests}

Some of the authors (SS, LZ, DT) are employed by the company which provided CNV-NT and the funding for this clinical trial.

\section{Publisher's Note}

Springer Nature remains neutral with regard to jurisdictional claims in published maps and institutional affiliations.

\section{Author details}

'Department of Veterinary Medicine and Surgery, Comparative Internal Medicine Laboratory, University of Missouri, College of Veterinary Medicine, 900 E. Campus Dr, Columbia, MO 65203, USA. ${ }^{2}$ Department of Veterinary Medicine and Surgery, Comparative Oncology Radiobiology and Epigenetics Laboratory, University of Missouri, College of Veterinary Medicine, $900 \mathrm{E}$. Campus Dr, Columbia, MO 65203, USA. ${ }^{3}$ Biomed Valley Discoveries, 4435 Main Street, Suite 550, Kansas City, MO 64111, USA.

Received: 12 September 2017 Accepted: 13 March 2018 Published online: 02 April 2018

\section{References}

1. Agrawal N, Bettegowda C, Cheong I, Geschwind JF, Drake CG, Hipkiss EL, Tatsumi M, Dang LH, Diaz LA Jr, Pomper M, et al. Bacteriolytic therapy can generate a potent immune response against experimental tumors. Proc Natl Acad Sci U S A. 2004;101(42):15172-7.

2. Roberts NJ, Zhang L, Janku F, Collins A, Bai RY, Staedtke V, Rusk AW, Tung D, Miller M, Roix J, et al. Intratumoral injection of Clostridium novyi-NT spores induces antitumor responses. Sci Transl Med. 2014;6(249):249ra111.

3. Krick EL, Sorenmo KU, Rankin SC, Cheong I, Kobrin B, Thornton K, Kinzler KW, Vogelstein B, Zhou S, Diaz LA Jr. Evaluation of Clostridium novyi-NT spores in dogs with naturally occurring tumors. Am J Vet Res. 2012;73(1): $112-8$.

4. Diaz LA Jr, Cheong I, Foss CA, Zhang X, Peters BA, Agrawal N, Bettegowda C, Karim B, Liu G, Khan K, et al. Pharmacologic and toxicologic evaluation of C. Novyi-NT spores. Toxicol Sci. 2005:88(2):562-75.

5. Axiak-Bechtel S, Fowler B, Yu DH, Amorim J, Tsuruta K, DeClue A. Chemotherapy and remission status do not alter pre-existing innate immune dysfunction in dogs with lymphoma. Res Vet Sci. 2014;97(2):230-7.

6. Fowler BL, Axiak SM, DeClue AE. Blunted pathogen-associated molecular pattern motif induced TNF, IL-6 and IL-10 production from whole blood in dogs with lymphoma. Vet Immunol Immunopathol. 2011;144(1-2):167-71.

7. LeBlanc CJ, LeBlanc AK, Jones MM, Bartges JW, Kania SA. Evaluation of peripheral blood neutrophil function in tumor-bearing dogs. Vet Clin Pathol. 2010;39(2):157-63.

8. Zhang Y, Axiak-Bechtel S, Friedman Cowan C, Amorim J, Tsuruta K, DeClue AE. Evaluation of immunomodulatory effect of recombinant human granulocyte-macrophage colony-stimulating factor on polymorphonuclear cell from dogs with cancer in vitro. Vet Comp Oncol. 2016;15(3):968-79.

9. Floras AN, Holowaychuk MK, Bienzle D, Bersenas AM, Sharif S, Harvey T, Nordone SK, Wood GA. N-terminal pro-C-natriuretic peptide and cytokine kinetics in dogs with endotoxemia. J Vet Intern Med. 2014;28(5):1447-53.

10. Karlsson I, Wernersson S, Ambrosen A, Kindahl H, Södersten F, Wang L, Hagman R. Increased concentrations of C-reactive protein but not highmobility group box 1 in dogs with naturally occurring sepsis. Veterinary Immunology and Immunopathology. 2013;156(1-2):64-72.

11. Filiz Ibraimi, Björn Ekberg, Dario Kriz, Gertrud Danielsson, Leif Bülow. Preparation of a portable point-ofcare in vitro diagnostic system, for quantification of canine C-reactive protein, based on a magnetic two-site immunoassay. Analytical and Bioanalytical Chemistry, 2013;405(18):60016007.

12. Deitschel SJ, Kerl ME, Chang CH, DeClue AE. Age-associated changes to pathogen-associated molecular pattern-induced inflammatory mediator production in dogs. J Vet Emerg Crit Care (San Antonio). 2010;20(5):494-502.

13. Yu D, Kim J, Park C, Park J. Serial changes of CD4+CD25+FoxP3+ regulatory T cell in canine model of sepsis induced by endotoxin. J Vet Med Sci. 2014; 76(5):777-80.

14. Maletzki C, Gock M, Klier U, Klar E, Linnebacher M. Bacteriolytic therapy of experimental pancreatic carcinoma. World J Gastroenterol. 2010;16(28): 3546-52.

15. Van Andel RA, Hook RR Jr, Franklin CL, Besch-Williford CL, van Rooijen N, Riley LK. Effects of neutrophil, natural killer cell, and macrophage depletion on murine Clostridium piliforme infection. Infect Immun. 1997;65(7):2725-31.

\section{Submit your next manuscript to BioMed Central and we will help you at every step:}

- We accept pre-submission inquiries

- Our selector tool helps you to find the most relevant journal

- We provide round the clock customer support

- Convenient online submission

- Thorough peer review

- Inclusion in PubMed and all major indexing services

- Maximum visibility for your research

Submit your manuscript at www.biomedcentral.com/submit
) Biomed Central 